\title{
Rítudes de matériaux susceptibles de résister à la corrosion par l'eau de mer
}

\author{
par J. Leğrand \\ Département Étude des Matériaux, \\ Direction des Études et Recherches, \\ Électricité de France.
}

\section{Introduction}

Les océans constituent une réserve biologique et un espace qui devront être exploités, dans les années à venir, pour résoudre les problèmes liés au développement démographique de la planète. L'exploitation 'de ces richesses ne fait que débuter mais, déjà, apparaissent de nombreuses réalisations utilisant les mers, soit comme source d'eau que l'on peut désaliniser, soit comme une source de frigories pour la réfrigération des usines de production d'électricité 'd'origine thermique, par exemple. En effet, les installations de centrales en bord de mer, et même de centrales flottantes aux U.S.A., se multiplient, ce qui constitue un aspect, parmi tant d'autres, du développement des techniques utilisant l'eau de mer.

Certaines difficultés technologiques apparaissent au cours de toutes ces réalisations, elles concernent, notamment, la tenue des matériaux en fonction de leurs conditions de service en milieu marin. L'usine de la Rance a été l'occasion de réaliser des progrès importants dans ce domaine ainsi que l'ont montré les exposés précédents. L'étude des propriétés des matériaux et de leurs conditions d'utilisation a été poursuivie depuis dix ans. Et nous désirons montrer, dans cet exposé, quelques exemples 'de progrès réalisés ou recherchés dans la connaissance des milieux marins et des techniques employées pour accroître la durabilité des installations.

\section{1. - Connaissance du milieu biologique marin et protections des installations contre les solissures}

Le milieu marin, milieu complexe mais équilibré, est constitué d'eau de mer, de gaz 'dissous, d'une flore, d'une faune et de particules solides en suspension.

La composition chimique de l'eau de mer du large (tableau 1) est d'une remarquable stabilité. Près des côtes, la salinité varie en raison d'apport d'eau douce, mais les proportions relatives des divers éléments restent les mêmes.

La présence d'oxygène et de gaz carbonique résulte d'échanges avec l'atmosphère et, localement, de réactions photosynthétiques, de l'action chlorophyllienne des plantes, de l'activité microbienne et animale. Leur concentration varie avec la température de l'eau. Près des côtes, dans les estuaires, dans les ports et les bassins, la vie des espèces marines est particulièrement intense, étant donné la grande fertilité des eaux. Les éléments nutritifs, nécessaires à leur vie, sont amenés en quantité par le mouvement des eaux assujetti aux conditions climatiques du continent. La prolifération de la flore et de la faune est donc saisonnière. Dans cet ensemble, microbes et bactéries ont un rôle de fossoyeurs et leur action libère des quantités non négligeables d'hydrogène sulfuré et d'ammoniaque.

L'Homme, par la pollution, prend une part active dans les modifications physico-chimiques du milieu marin. Ses rejets peuvent accroître la fertilité des eaux, ou les rendre toxiques, ou même très agressives pour certaines installations. On peut citer, à titre d'exemple, les cas 'de corrosion du condenseur de la centrale électrique de Dunkerque. Entre les essais préliminaires dans un condenseur expéri- 


\begin{tabular}{|c|c|c|}
\hline \multicolumn{2}{|c|}{$\begin{array}{l}\text { Composition chimique de l'eau de mer } \\
\text { en pour } 1000 \text { ou grammes par litre. }\end{array}$} & Tableau 1 \\
\hline Cations & $\begin{array}{l}\mathrm{Na}_{+}^{+} \\
\mathrm{Mg}^{++} \\
\mathrm{Ca}^{++}+ \\
\mathrm{K}+ \\
\mathrm{Sr}^{++}\end{array}$ & $\begin{array}{c}10,77 \\
1,30 \\
0,409 \\
0,338 \\
0,010\end{array}$ \\
\hline Anions & $\begin{array}{l}\mathrm{Cl}^{-} \\
\mathrm{SO}_{4}^{--}- \\
\mathrm{HCO}_{3}- \\
\mathrm{Br}^{-} \\
\mathrm{F}^{-}\end{array}$ & $\begin{array}{c}19,37 \\
2,712 \\
0,142 \\
0,065 \\
0,001\end{array}$ \\
\hline \multicolumn{2}{|c|}{ Acide borique } & 0,026 \\
\hline \multicolumn{2}{|c|}{$\begin{array}{l}\text { Oligoéléments : } \\
\left(\mathrm{Cu}, \mathrm{Zn}, \mathrm{Mn}, \mathrm{NO}_{3}^{-}, \mathrm{PO}_{4}^{---}\right)\end{array}$} & traces \\
\hline
\end{tabular}

Classification des métaux et alliages selon leur potentiel de dissolution dans

l'eau de mer en mouvement et saturée en oxygène

\begin{tabular}{|c|c|}
\hline Métaux et alliages & $\begin{array}{c}\text { POTENTIEL } \\
\text { DE DISSOLuTION } \\
\text { (en mV/e.c.s.) }\end{array}$ \\
\hline Magnésium & -1500 \\
\hline Zinc & -1030 \\
\hline Aluminium & -790 \\
\hline Cadmium & -700 \\
\hline Acier & -610 \\
\hline Acier inoxydable $18 / 8$ actif & $-\quad 530$ \\
\hline Plomb & -500 \\
\hline Fonte Ni resist & -470 \\
\hline Etain & $-\quad 420$ \\
\hline Laiton marine & -400 \\
\hline Cuivre & $-\quad 360$ \\
\hline Gunmetal (Cu-Sn-Zn) & $-\quad 310$ \\
\hline Laiton amirauté & -290 \\
\hline Cupro-aluminium & -290 \\
\hline Cupro-nickel $90 / 10$ & -280 \\
\hline$-\quad 80 / 20$ & -270 \\
\hline$-\quad 70 / 30$ & -250 \\
\hline Nickel & $-\quad 220$ \\
\hline Nickel cuivre 400 & $-\quad 110$ \\
\hline Titane & -100 \\
\hline Acier inoxydable $18 / 8$ passif & +80 \\
\hline Graphite & +250 \\
\hline Platine & +260 \\
\hline
\end{tabular}

mental, ayant permis de sélectionner les tubes en laiton à l'aluminium, et leur utilisation dans le condenseur de la centrale, l'intensification du trafic des pétroliers, qui manœuvrent devant la prise d'eau, a augmenté la quantité de sable en suspension, provoquant ainsi la corrosion-érosion des tubes. De plus, l'élévation locale de la température a favorisé le développement d'espèces marines tels les moules et les tarets. Des études sont actuellement en cours, notamment au Centre de Recherches et d'Etudes Océanographiques (C.R.E.O.), et ont pour but, soit la connaissance des différentes espèces d'algues, de leur répartition géographique et des conditions dans lesquelles elles apparaissent, ainsi que la mise au point d'indicateurs de pollution, et la détermination de l'action polluante due aux produits antisalissures.

Selon leur nature et leur densité, les salissures engendrent des modifications locales de composition chimique du milieu. Leur fixation peut s'opposer ou contrarier l'écoulement de veines liquides. Leur entraînement, par une veine liquide circulant à grande vitesse, peut causer des phénomènes de corrosion-érosion.

Les moyens actuels de protection contre les salissures répondent tous à des applications précises. Ils se divisent en :

\section{Moyens physiques:}

Une filtration en amont de circuit d'alimentation est opérée à l'aide de systèmes grilles fixes-dégrilleurs, de grilles rotatives et de filtres spéciaux. Un nettoyage mécanique est réalisé périodiquement dans les canaux d'amenée (raclage), ou de façon continue dans les tubes de condenseurs (circulation des sphères en caoutchouc mousse).

N.B. - On notera que les divers systèmes de filtration sont sans effet contre les microorganismes, qui peuvent se fixer et se développer sur les parois des boîtes à eau de condenseur, et leur décrochement massif ultérieur éventuel qui peut obturer de nombreux tubes.

\section{Moyen thermique:}

Un échauffement de l'eau de mer (recirculation de l'eau de refroidissement ou apports de vapeur) modifie l'écologie marine et les moules notamment ne résistent pas à un maintien d'une heure à $40^{\circ} \mathrm{C}$.

\section{Moyens chimiques:}

Certaines installations (installations portuaires fixes, carènes de navires...) se prêtent à la protection anti-salissures par l'application de peintures ou enduits toxiques. Pour d'autres (condenseurs de centrales électriques...) on procède à des injections de chlore.

Les peintures et enduits toxiques sont des produits à base de cuivre surtout, de mercure, d'arsenic et autres... Leur effet est lié à leur dissolution qui crée, le long des surfaces à protéger, des zones toxiques empêchant l'accrochage des salissures. Ces produits, non hydrofuges, doivent être appliqués sur des peintures isolantes garantissant les substrats contre un éventuel effet galvanique dû à la présence du cuivre, en général. Du fait de leur mode d'action, l'efficacité de ces produits est limitée dans le temps. Elle dépend de la nature des salissures et du degré d'éclairement de l'eau.

La chloration de l'eau de mer vise à empêcher la prolifération des organismes vivants. C'est l'un des procédés les plus employés tant en France qu'à l'étranger. Sa mise au point est délicate. Le taux de chlore injecté en début de 
circuit conduit à une teneur résiduelle, en cet élément, inférieure à $0,2 \mathrm{ppm}$ à l'entrée du condenseur. L'accoutumance des organismes marins, moules notamment, à une injection discontinue mais périodique, a conduit la Production Thermique d'E.D.F. à décider de la chloration en continu du $1^{\text {rr }}$ mars au $1^{* r}$ novembre pour la centrale de Dunkerque. Le chlore employé est liquéfié et sous pression ( 5 bars à $10^{\circ} \mathrm{C}$ ). Pour des raisons de sécurité, il peut être remplacé par de l'eau de javel, mais cette substitution triple le prix de revient de l'opération. Un autre procédé consiste à produire le chlore selon les besoins en électrolysant une partie de l'eau de mer.

\section{Moyens piézo-électriques et électro-chimiques:}

Dans des petites installations industrielles et en laboratoire, des résultats intéressants ont été notés avec la protection par ultra-sons. La protection cathodique peut avoir un rôle bénéfique (exemple les groupes de la Rance). La forte alcalinisation, qui se produit au niveau des surfaces protégées, peut être la cause d'une non incrustation ou d'une désincrustation selon le cas. Mais, lorsque des densités de courant élevées sont nécessaires, elle peut rendre le procédé dangereux pour les revêtements de peinture. Une étude approfondie de l'effet des courants électriques sur les organismes marins est en cours au laboratoire d'électro-physiologie de la Station d'Hydrologie de Biarritz, avec la collaboration d'E.D.F. L'action paralysante du courant électrique et des ultra-sons sur les larves 'doit permettre d'empêcher leur fixation dans les condenseurs de centrales électriques.

\section{2. - Propriétés électro-chimiques de l'eau de mer}

L'eau de mer est un électrolyte de faible résistivité (20 à $30 \Omega / \mathrm{cm})$. La corrosion des matériaux pourra prendre différents aspects qui dépendront de leur potentiel de dissolution, de leur tendance à la passivité, de leurs conditions d'utilisation (couplage galvanique avec d'autres métaux, présence de crevasses, d'interstices, phénomènes d'érosion...). L'oxydation des métaux est équilibrée cathodiquement par la réduction de l'oxygène dissous ou, pour les métaux très électro-négatifs, par la réduction des protons. Un premier classement de la résistance des matériaux à la corrosion par l'eau de mer peut se faire par une mesure de son potentiel de dissolution (tableau 2). Depuis plusieurs années, le développement des techniques d'étude électro-chimique de la corrosion permet de prévoir leur comportement dans l'eau de mer. Le tracé des courbes intensité-potentiel de chaque matériau à l'aide de potentiostats montre:

- Le risque de corrosion par couplage des matériaux et la corrosion qui en résultera. Le courant anodique du métal le plus électro-négatif sera équilibré par la réduction cathodique de l'oxygène ou de l'ion hydrogène sur l'autre métal. La corrosion, par différence d'aération dans les interstices dans les joints soudés, boulonnés ou rivetés, n'est qu'un aspect de cette corrosion galvanique.

- Le risque de corrosion par piqûres qui affecte de nombreux métaux passivables. On note, sur ces courbes, un potentiel de protection au-delà duquel il y a risque de piqûres.
- Le degré de sensibilisation à la corrosion intergranulaire de certains alliages. L'application de cette méthode aux aciers inoxydables avait constitué l'une des premières utilisations de ces courbes aux potentiostats par Edéléanu. Cette technique a permis, également, de mesurer la susceptibilité à ce type de corrosion des alliages d'aluminium à 5 et $7 \%$ magnésium laminés.

\section{3. - Tendances actuelles de protection de l'acier}

L'acier, fortement corrodable par l'eau de mer, doit être protégé. Les méthodes de protection adoptées sont, soit la protection cathodique traitée par ailleurs, soit la réalisation de revêtements, isolant le subjectile du milieu, ou soit une combinaison de ces deux méthodes.

Les revêtements par peinture se sont imposés économiquement pour les carènes de navires. L'utilisation d'hélice en matériaux nobles a posé un problème de corrosion des poupes, résolu par la mise en place d'anodes solubles en zinc ou en magnésium. L'extension de la protection cathodique, sur tout le pourtour d'un bateau, renforce la durabilité des peintures. Actuellement, les unités de la Marine Nationale passent en carénage tous les deux ans. Les systèmes de peinture appliquiés sont constitués:

- soit d'un wash primer (butyro-chlorure de vinyl ou chromate de zinc), de couches isolantes pur brai, entre des couches de brai pigmenté à l'aluminium en paillettes et d'un antifouling (l'ensemble représentant une épaisseur de 150 microns);

- soit d'un wash primer, de couches de brai aux résines époxydiques et d'un antifouling appliqué à chaud (l'ensemble représentant une épaisseur de 250 microns).

Le carénage consiste, bien souvent, en la réfection des couches de peintures toxiques. Pour les installations portuaires, on utilise plus couramment des peintures riches en zinc, conçues pour apporter une protection du même type que celle offerte par les revêtements de zinc. Des portes d'écluses, dans le port de Rouen, sont revêtues d'un primaire époxy-zinc, recouvert de peintures à base de résines époxydiques. Dans le port autonome de Dunkerque, les portes de l'écluse Charles-de-Gaulle sont protégées par 40 microns d'époxy-zinc et 500 microns de brai aux résines époxydiques.

Dans certaines centrales électriques, les boîtes à eau en acier sont protégées, soit par placage de feuille de néoprène, soit par plusieurs couches de néoprène, soit par peintures à base de caoutchouc ou de résines époxydiques ou de polyuréthane.

Mentionnons l'utilisation, de plus en plus fréquente, sur les chantiers navals notamment, de tôles et profilés grenaillés et prépeints. Cette possibilité est actuellement à l'étude à E.D.F.

L'acier peut, avantageusement, être protégé par un revêtement métallique anodique (zinc, aluminium). Mais, pour éviter sa dissolution trop rapide dans l'eau de mer et pour boucher ses pores et micropores inévitables, il convient de procéder à l'application de couches de peinture isolante et de nature compatible avec lui. Cette protection est une solution onéreuse mais qui permet de procéder aux opérations d'entretien dans de bien meilleures conditions. De plus, 
comme toutes les peintures finissent par se détremper, le métal anodique pourra alors jouer son rôle et permettre d'atteindre la période d'entretien prévue. Certains tuyautages, par exemple, sont en acier galvanisé et revêtus d'asphalte, de bitume, de brai aux résines époxydiques, de ciment. Des installations portuaires, telle qu'une porte de l'écluse Vatier, dans le port de Dunkerque, sont métallisées au zinc et revêtues de peinture riche en zinc. De même, quelques petites unités de pêche (chalutiers) ont subi un traitement analogue.

A ce sujet, il nous faut mentionner une étude, entreprise sous l'égide du Centre Français de la Corrosion, concernant la protection de l'acier par métallisation et peinture. Des éprouvettes en acier, constituées d'une plaque et d'une cornière boulonnées, ont été métallisées au zinc $(40,80,120$, 160 et $200 \mu)$, à l'aluminium $(120,200$ et $300 \mu)$, et aux alliages zinc-aluminium de compositions pondérales $85 / 15$ et $60 / 40(40,80$ et $120 \mu)$ et revêtues d'un système de peinture cohérent pris dans chacune des quatre feuilles suivantes: vinylique, polyuréthane, brai aux résines époxydiques et brai de houille. Ces éprouvettes sont immergées, depuis plus de cinq années, dans des stations d'immersion de l'E.D.F. et de l'I.R.S.I.D. Les résultats essentiels ont été publiés lors des dernières journées du C.E.F.R.A.C.O.R.

Il est apparu qu'une métallisation à l'alliage zinc-aluminium $(85 / 15)$ présentait des qualités excellentes et, que revêtue de brai aux résines époxydiques, elle constituait l'une des solutions mixtes les plus satisfaisantes dans l'eau de mer, aussi bien à la station d'E.D.F. de Lavera, qu'à celle de l'I.R.S.I.D. à Biarritz.

\section{4. - Choix des matériaux métalliques Matériaux nouveaux}

Le tableau 3 indique les compositions chimiques des principaux matériaux utilisés dans l'eau de mer, ainsi que quelques exemples de leurs applications. Quelques remarques s'imposent néanmoins et concernent :

\subsection{Les laitons.}

Les laitons monophasiques sont inhibés contre la dézincification par une addition d'arsenic. Ce n'est pas le cas des laitons à deux phases qui restent sensibles à ce phénomène. De ce fait, le métal Muntz est de plus en plus remplacé par un alliage de cuivre et d'aluminium pour les plaques de condenseur.

Le laiton à l'aluminium est une solution économique satisfaisante pour des tubes de condenseurs, refroidis par de l'eau de mer propre, et ne circulant pas à plus de $2.5 \mathrm{~m} / \mathrm{s}$. Dans de l'eau de mer polluée, ou pour des vitesses supérieures, leur substitution par des tubes en alliage de cuivre et de nickel est recommandée. La composition chimique de ces derniers doit contenir du fer, notamment, qui assure une meilleure protection. Notons également qu'une protection chimique de ces tubes peut être réalisée par production ou injection de sel ferreux dans l'eau de réfrigération. Le mode d'action de ce sel est encore mal connu, mais il se traduit par le dépôt d'oxydes ferriques sur les tubes protégés.

\subsection{Les alliages légers.}

Ceux mentionnés dans le tableau ont un bon comportement, mais sont sensibles à la corrosion galvanique dans les assemblages avec de nombreux autres matériaux.

\subsection{Les cupro-cluminiums.}

L'expérience a montré que ces matériaux pouvaient être le siège d'une corrosion sélective de la phase $\alpha$ de l'aluminium. Des études récentes ont défini que la désaluminisation n'était plus à craindre si la teneur en aluminium est inférieure à $8,5+(\% \mathrm{Ni} / 2)$, si les traitements thermiques ne stabilisent pas de phase $\beta$ massive et si les conditions d'utilisation ne font pas apparaître des différences de vitesse de l'eau en contact avec le matériau.

\subsection{I.es aciers inoxydables.}

Les aciers inoxydables austénitiques, du type $18 / 8$ ou $18 / 10$ au molybdène, peuvent, sous certaines conditions, subir des graves dommages par corrosion caverneuse ou par piqûres.

Dans la mesure où la géométrie d'un ensemble présente des risques de crevasses, où la répartition de l'oxygène ne sera pas homogène, on a intérêt à utiliser un acier austénitique au chrome-nickel-molybdène, plus chargé en nickel et en molybdène, que l'acier de type $18 / 12 / 3$, bien que celui-ci ait un bon comportement dans l'eau de mer oxygénée et en mouvement. Les nuances de remplacement pourraient être $18 / 14 / 3$ ou $18 / 16 / 6 / 3$ (à $3 \%$ de cuivre).

Pour des matériels soumis à des sollicitations mécaniques intenses, les aciers austénitiques sont insuffisants et, souvent, on a utilisé un acier martensitique, par exemple du type $17 / 1$ au molybdène, comme cela a été le cas pour les pales des roues et des aubes directrices des groupes de la Rance, qui donne lieu à des incrustations et des défauts en période d'arrêt prolongé (voir exposé de M. Leborgne). Les aciers martensitiques seraient, avantageusement, remplacés par des aciers austénoferritiques qui présentent une meilleure résistance à la corrosion, en général, tout en conservant des caractéristiques mécaniques acceptables et une bonne aptitude au moulage et au soudage. Mentionnons quelques compositions courantes de ces nuances: le 20/5/3, le $18 / 8 / 3 / 1,5$, le $23 / 4 / 3 / 1,5$, le $21 / 8 / 3 / 1,5$ et le $25 / 8 / 4 / 1,5$, les quatre dernières nuances contenant du cuivre en plus du chrome, du nickel et du molybdène.

On connaît l'effet favorable du chrome dans l'inaltérabilité des alliages fer-chrome. Les aciers ferritiques, contenant 26 à $30 \%$ de chrome, et de très faibles teneurs en carbone $(<0,003)$ et en azote $(<0,015)$, sont pratiquement insensibles à la corrosion intergranulaire, à la corrosion par piqûres et à la corrosion sous tension. Cependant, leur essor industriel a, pendant très longtemps, été contrarié par une certaine fragilité et leur extrême sensibilité à l'effet d'entaille. L'amélioration de leur résilience a été obtenue en abaissant les teneurs en carbone et en azote aux valeurs précitées. Ce matériau, très ductile, est envisagé pour des canalisations, échangeurs et condenseurs.

A côté de ces matériaux, dans l'ensemble, déjà éprouvés en eau de mer, il faut mentionner le développement nouveau de plusieurs métaux ou alliages, réputés chers, mais qui peuvent présenter, néanmoins, un grand intérêt. Il s'agit des alliages austénitiques à haute teneur en nickel et du titane. 
Tableau 3

Principaux alliages utilisés dans l'eau de mer

\begin{tabular}{|c|c|c|}
\hline DÉNOMINATIONS & $\begin{array}{l}\text { COMPOSITIONS CHIMIQUES } \\
\qquad(\mathrm{en} \%) \\
\end{array}$ & TYPES D'APPLICATIONS \\
\hline Acier revêtu & & $\begin{array}{l}\text { Tuyautages, installations portuaires, carènes, boîtes à } \\
\text { eau... }\end{array}$ \\
\hline Fonte à graphite sphéroïdal & & Tuyautages, corps de pompes, vannes, boîtes à eau... \\
\hline Fonte austénitique & $\mathrm{Ni}>13$ & Corps de pompes, corps de vannes, boîtes à eau... \\
\hline Acier austénitique & $\left|\begin{array}{l}\mathrm{C} 0,02-\mathrm{Cr} 18-\mathrm{Ni} 12-\mathrm{Mo} 3-\mathrm{Cu} 0,5 \\
\mathrm{C} 0,07-\mathrm{Cr} 18-\mathrm{Ni} 12-\mathrm{Mo} 3-\mathrm{Ti} 0,40\end{array}\right|$ & Impulseurs de pompes, câbles, citernes, cuves, tubes... \\
\hline Acier martensitique & $\begin{array}{l}\text { Cr } 17-\mathrm{Ni} 4-\text { Mo } 1,5 \\
\text { Cr } 16-\text { Ni } 2 \text { - Mo } 1,5\end{array}$ & Axes de pompes, hélices, turbines, boulonnerie... \\
\hline Acier ferritique & $\mathrm{Cr} 15$ à $30-\mathrm{C}$ & Pièces forgées ou moulées. \\
\hline Acier austéno-ferritique & $\begin{array}{c}\mathrm{C} \leqslant 0,05-\operatorname{Cr} 2 \mathrm{I}-\mathrm{Ni} 8-\mathrm{Mn} \leqslant 1,5 \\
\text { Mo } 2,5-\operatorname{Cu} 1,5\end{array}$ & $\begin{array}{l}\text { Pièces moulées de préférence pales d'hélice, de roue } \\
\text { de turbine... }\end{array}$ \\
\hline Ferrite au chrome & $C \leqslant 0,003-\operatorname{Cr} 26 / 30-N \leqslant 0,015$ & Tubes d'échangeurs. \\
\hline Cuivre & & Tuyautages (vitesse $<0,4 \mathrm{~m} / \mathrm{s}$ ). \\
\hline Laiton à l'aluminium & $\mathrm{Cu} 76-\mathrm{Zn} 22-\mathrm{Al} 2-\mathrm{As} 0,1$ & Tubes d'échangeurs. \\
\hline Métal Muntz & $\mathrm{Cu} 60-\mathrm{Zn} 40$ & Plaques à tubes. \\
\hline Laiton marine & $\mathrm{Cu} 60-\mathrm{Zn} 39-\mathrm{Sn} 1$ & Plaques à tubes. \\
\hline Bronze $\mathrm{G}$ & $\mathrm{Cu} 88-\mathrm{Sn} 10-\mathrm{Zn} 2$ & Corps de pompes et de vannes, impulseurs. \\
\hline Cupro-aluminium 1 & $\mathrm{Cu} 87-\mathrm{A} 19-\mathrm{Ni} 2-\mathrm{Fe} 2-\mathrm{Mn} 1,5$ & $\begin{array}{l}\text { Pompes, hélices, plaques à tubes, tuyautage, vannes, } \\
\text { boulonnerie... }\end{array}$ \\
\hline Cupro-aluminium 2 & $\mathrm{Cu} 81,5-\mathrm{Al} 9-\mathrm{Ni} 5-\mathrm{Fe} 3-\mathrm{Mn} 1,5$ & $\begin{array}{l}\text { idem (meilleure résistance à la cavitation et à la cor- } \\
\text { rosion sous tension). }\end{array}$ \\
\hline Cupro-nickel 90/10 & $\mathrm{Cu} .90-\mathrm{Ni} 10-\mathrm{Fe} 1,5-\mathrm{Mn} 1$ & Tubes d'échangeur. \\
\hline Cupro-nickel $70 / 30$ & 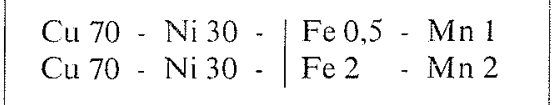 & Tubes d'échangeur. \\
\hline Alliage léger AG4MC & $\mathrm{Mg} 4 \cdot \mathrm{Mn} 0,4 \cdot \mathrm{Cr} 0,2$ & Carènes de petits navires. \\
\hline Alliage léger AG6 & $\operatorname{Mg} 6$ & Pièces moulées de formes complexes. \\
\hline Monel 400 & $\mathrm{Ni} 94-\mathrm{C} \leqslant 0,3-\mathrm{Mn} 2-\mathrm{Fe} 2,5-\mathrm{Si} 0,5$ & $\begin{array}{l}\text { Soupapes, pompes, arbres de pompes et d'hélices, } \\
\text { impulseurs, tubes. }\end{array}$ \\
\hline Monel K 500 & $\begin{array}{c}\text { Ni } 90-\mathrm{C} \leqslant 0,3-\mathrm{Mn} 2-\mathrm{Fe} 2-\mathrm{Si} 1 \\
\text { Al } 2 \text { à } 4-\mathrm{Ti} 0,25-1\end{array}$ & Arbres, rotors. \\
\hline
\end{tabular}




\section{- Les alliages de nickel.}

L'inconel 625, outre le nickel, contient 20 à $23 \%$ de chrome, 8 à $10 \%$ de molybdène, moins de $5 \%$ de fer, du niobium et du tantale à raison de 3,15 à $4,15 \%$. Il allie à une bonne tenue mécanique, une grande facilité de mise en œuvre, soudage notamment, et une excellente résistance aux divers aspects signalés de la corrosion. Aucun effet de fragilisation n'est à craindre et un traitement thermique après soudage ne s'impose pas à l'alliage, qui conserve résistance et ductilité.

Ses applications principales sont : fils de câbles d'amarrage et gaine de câble de communication, pales d'hélices, moteurs auxiliaires de propulsion, moteurs de pompes, composants d'instruments océanographiques, boulonnerie de sécurité, etc.

L'hastelloy $\mathrm{C}$ est, comme l'inconel, un alliage de nickel. Il contient, en outre, $16 \%$ de chrome, 15 à $18 \%$ de molybdène, 5 à $6 \%$ de fer et, parfois, du tungstène. La forte teneur en molybdène peut provoquer une certaine fragilité, d'où une certaine sensibilité à la fissuration dans les zones de soudure et à la corrosion intergranulaire. Ce phénomène est atténué fortement en maintenant la teneur de carbone à une valeur très basse. Sa résistance à la corrosion est remarquable, il trouve son utilisation dans certaines conditions sévères pour lesquelles les aciers austénitiques, même chargés en nickel et en cuivre, sont insuffisants.

\section{Le titane.}

La demande croissante de titane, pour l'aéronautique et l'espace, a créé des conditions économiques telles que l'utilisation de ce métal, pour des applications plus courantes, est actuellement envisageable grâce à sa grande faisabilité, notamment pour les faisceaux tubulaires de condenseurs refroidis par l'eau de mer.

Son excellent comportement dans l'eau de mer, sous diverses conditions, autorise des réductions d'épaisseur efficace. Malgré un coût plus élevé, l'amincissement du tube en titane le rend compétitif avec des tubes en cupro-alliages de même diamètre extérieur. Ainsi, un condenseur entièrement équipé en tubes en titane serait moins cher que s'il était équipé en tubes de cupro-nickel 70/30, compte tenu des différentes modifications de forme consécutives aux propriétés du titane (coefficient d'échange thermique, résistance à l'érosion...).

Des essais de corrosion par l'eau de mer, de tubes en titane non alliés, roulés et soudés ou étirés sans soudure, sont en cours dans notre station de corrosion de Lavera. La vitesse de circulation de l'eau est réglée, par diaphrag. mes, à 2 et $3 \mathrm{~m} / \mathrm{s}$. Les résultats des premières observations, effectuées après plus de 10000 heures de fonctionnement, confirment la bonne tenue du matériau. Les parois internes des tubes se recouvrent seulement d'une pellicule de boue biologique non adhérente.

\section{Conclusion}

Nous avons cherché à montrer, dans cet exposé, quelques exemples des études nouvelles et des progrès réalisés dans le domaine de la corrosion marine : connaissance du milieu marin et des salissures engendrant la corrosion, technique d'étude, protection des structures en acier et développement de nouveaux métaux et alliages.

Il faut bien reconnaître, néanmoins, que les progrès réalisés visent d'autres applications que la construction d'usines marémotrices, et que l'excellent fonctionnement de la protection cathodique dans le cas de la Rance, tel que l'ont décrit les exposés précédents, semble suffisamment éprouvé pour éviter de rechercher des solutions radicalement nouvelles si un projet semblable se présentait aujourd'hui.

\section{Discussion}

président: M. P. CaBaniols

M. le Président remercie M. Legrand et ouvre la discussion.

Les métaux à fort pourcentage de nickel et de chrome, remarque M. Leborgne, ont souvent des limites de fatigue assez faibles; on peut rappeler le cas de l'acier "Uranus 50 » ayant une teneur en chrome supérieure à $20 \%$ et qui a donné lieu, sur des roues Pelton, à des ruptures par fatigue. Connaissez-vous la limite de fatigue de ces métaux, tel que l'inconel et l'hastelloy que vous citez dans votre mémoire?

Nous n'avons pas entrepris d'études dans ce domaine, répond M. Legrand, mais le fait que ces matériaux soient couramment utilisés sur les navires de la marine militaire aux Etats-Unis semble prouver que leur résistance à la fatigue est satisfaisante.

M. Heuzé (Société d'Etudes contre la Corrosion, Paris) s'interroge sur un éventuel effet anti-fouling de la protection cathodique. II signale que M. Benedict de la Société Lockheed a constaté que la protection cathodique n'avait aucun effet - positif ou négatif - sur les salissures. Il faut donc bien préciser que c'est le courant électrique qui contrarie la vie animale et que c'est seulement sur les métaux localement ou complètement nus que la protection cathodique est susceptible d'avoir un effet anti-fouling.
M. Legrand rappelle les observations signalées dans l'exposé précédent de M. LeBorgne: des incrustations très importantes, des dépôts d'algues et de moules ont été observés sur des aciers du type 17.4, notamment sur les pales des turbines de la Rance, lorsque ces pièces étaient maintenues immergées immobiles sans protection cathodique; lorsque l'ensemble a été mis sous protection cathodique, on n'a plus observé ces incrustations ou ces dépôts d'algues.

M. LAMBERT donne son opinion en ces termes:

«Quand on parle d'anti-fouling, il s'agit, en général, d'un complément à un système de peinture pour éviter la fixation d'organismes sur cette peinture, et en cette matière, je rejoins le propos de M. Heuzé; je ne pense pas que la protection cathodique envisagée sous cet angle ait un effet anti-fouling. En fait l'effet anti-fouling constaté est lié au passage du courant électrique, c'est-à-dire que le courant, sur des pièces métalliques en contact avec l'eau de mer, empêche le développement des êtres vivants; sur un métal nu la protection cathodique peut être considérée comme ayant une action anti-fouling; le revêtement de peinture, à l'endroit où il est en bon état, par sa résistance d'isolement électrique s'oppose au passage du courant, il n'y a donc aucune raison pour que la protection cathodique sur la peinture ait un effet anti-fouling. » 
Se référant à l'exposé de M. LEgRAND concernant les systèmes 《revêtements-métaux » compatibles, il poursuit:

«Un revêtement de peinture peut être considéré comme une fin en soi si l'on a affaire à un ensemble homogène de pièces métalliques, par exemple en acier ordinaire, parce que les défauts inévitables sur ce genre de revêtement ne conduiront qu'à mettre à nu une fraction déterminée de métal qui se dissoudra à sa vitesse d'auto-corrosion classique et naturelle. Par contre, dans le cas d'ensembles polymétalliques l'isolement d'une fraction importante de l'acier ordinaire, s'il se trouve relié électriquement à un métal plus noble, fait que dès lors la polarisation anodique qu'il va recevoir en l'absence de protection cathodique au droit des défauts augmentera considérablement la vitesse de corrosion; dans ces cas-là, la protection cathodique est pratiquement indispensable. C'est ce qu'indique M. Legrand au sujet des protections de condenseurs: les revêtements de peinture à l'intérieur des boîtes de condenseurs ne font qu'augmenter la vitesse de corrosion là où l'acier ordinaire est en contact avec l'eau de mer du fait de l'influence des plaques en cupro-aluminium voisines, 》

Personne ne demandant plus la parole, $M$. le Président conclut la séance et aussi la Session "Six ans d'exploitation de l'usine marémotrice de la Rance » par l'allocution suivante:

«Je remercie d'abord les auteurs des communications, ceux des interventions qui ont animé les discussions et tous les participants et en particulier les plus fidèles.

«Je pense que ces deux jours vous auront convaincus d'un certain nombre de choses. D'abord, sans vouloir pousser un «cocorico», on peut affirmer que l'usine de la Rance a été une réussite technique, aussi bien au stade des études qu'à celui de la réalisation, réalisation du génie civil et du matériel électromécanique, et aussi, finalement, à celui de l'exploitation. Les groupes, on vous l'a dit, ont une excellente "disponibilité ». Cela est essentiel dans une usine où il n'y a pas de basses eaux, à moins que l'on qualifie ainsi les marées de morte-eau, et, en effet, les exploitants s'attachent avec succès à avoir le maximum de groupes disponibles pour les marées de vive-eau.

Je crois qu'on peut dire aussi que la technique des groupes bulbes, qui a été certainement contestée à son apparition, et peut-être surtout par les futurs exploitants, a été un succès, un succès en soi et un succès par ce qu'elle a permis de réaliser par la suite.

M. Cotillon vous a cité ce matin le nombre de groupes de grandes dimensions en service ou qui vont être mis prochainement en service en France et je pense qu'il s'y en ajoutera beaucoup d'autres à l'étranger, parce qu'en France, malheureusement, nous voyons s'épuiser les gisements hydroélectriques.

Enfin, les études qui ont dû être faites pour lutter efficacement contre la corrosion ont été, elles aussi, couronnées de succès. $\mathrm{Ce}$ n'était pas non plus évident, et je me souviens que les futurs exploitants que nous étions avaient un peu froid dans le dos quand a été découvert l'état dans lequel se trouvait un groupe resté pendant plusieurs mois en eau stagnante à la Rance: les photos que M. Leborgne a fait projeter tout à l'heure vous ont montré dans quel état on les avait trouvés.

L'expérience acquise a déjà servi ailleurs qu'à la Rance, notamment à Martigues, à Dunkerque, à Cordemais, et je crois qu'elle servira encore dans toutes les installations en milieu marin, et spécialement dans les futures centrales nucléaires qui y trouveront l'eau de refroidissement qu'il devient si difficile d'utiliser ailleurs, même si elle existe. 